University of Nebraska - Lincoln

DigitalCommons@University of Nebraska - Lincoln

Civil Engineering Faculty Publications

Civil Engineering

December 2004

\title{
Efficient Algorithm for Computing Einstein Integrals
}

Junke Guo

University of Nebraska - Lincoln, jguo2@unl.edu

Pierre Y. Julien

Colorado State University, pierre@engr.colostate.edu

Follow this and additional works at: https://digitalcommons.unl.edu/civilengfacpub

Part of the Civil Engineering Commons

Guo, Junke and Julien, Pierre Y., "Efficient Algorithm for Computing Einstein Integrals" (2004). Civil Engineering Faculty Publications. 4.

https://digitalcommons.unl.edu/civilengfacpub/4

This Article is brought to you for free and open access by the Civil Engineering at DigitalCommons@University of Nebraska - Lincoln. It has been accepted for inclusion in Civil Engineering Faculty Publications by an authorized administrator of DigitalCommons@University of Nebraska - Lincoln. 


\title{
Efficient Algorithm for Computing Einstein Integrals
}

\author{
Junke Guo ${ }^{1}$ and Pierre Y. Julien²
}

\begin{abstract}
Analytical approximations to Einstein integrals are proposed. The approximations represented by two fast-converging series are valid for all values of their arguments. Accordingly, the algorithm can be easily incorporated into professional software like $H E C-R A S$ or HEC-6 with minimum computational effort.
\end{abstract}

DOI: 10.1061/(ASCE)0733-9429(2004)130:12(1198)

CE Database subject headings: Bed load; Suspended load; Sediment transport; Computation; Algorithms.

\section{Introduction}

The Einstein bed load function is a landmark of modern sediment transport mechanics. It provides the first theoretical framework for sediment transport calculation, which guided many of the following researchers. Nevertheless, the computation of Einstein bed load function requires an estimation of two integrals $J_{1}$ and $J_{2}$, which cannot be integrated in closed form for most cases and are very slowly convergent for direct numerical integration because of singularity of the integrands near the bed (Nakato 1984). Einstein (1950) provided a numerical table and graphs to facilitate the calculation. Some mathematical software, such as MatLab and Maple can also be used to integrate them numerically. However, both methods cannot be easily implemented in professional software. For example, the widely used HEC-RAS and HEC-6 do not include Einstein bed load function (U.S. Army Corps of Engineers 1993, 2003) probably because of the complexity. The purpose of this article is to provide a fast-converging algorithm to estimate Einstein integrals $J_{1}$ and $J_{2}$.

\section{Einstein Integrals}

In his bed load function, Einstein (1950) defined

$$
J_{1}(z)=\int_{E}^{1}\left(\frac{1-\xi}{\xi}\right)^{z} d \xi
$$

and

${ }^{1}$ Dept. of Civil Eng., Univ. of Nebraska-Lincoln, 205C PKI, $1110 \mathrm{~S}$. 67th St., Omaha, NE 68182; and, Affiliate Faculty, State Key Lab of Water Resources and Hydropower Eng. Sci., Wuhan Univ., Hubei 430072, PRC.

${ }^{2}$ Professor, Engineering Research Center, Dept. of Civil Engineering, Colorado State Univ., Fort Collins, CO 80523. E-mail: pierre@engr.colostate.edu

Note. Discussion open until May 1, 2005. Separate discussions must be submitted for individual papers. To extend the closing date by one month, a written request must be filed with the ASCE Managing Editor. The manuscript for this technical note was submitted for review and possible publication on February 3, 2003; approved on May 28, 2004. This technical note is part of the Journal of Hydraulic Engineering, Vol. 130, No. 12, December 1, 2004. CASCE, ISSN 0733-9429/2004/12$1198-1201 / \$ 18.00$.

$$
J_{2}(z)=\int_{E}^{1}\left(\frac{1-\xi}{\xi}\right) z \ln \xi d \xi
$$

where $E=$ relative bed-layer thickness to water depth. Eq. (1) originates from Rouse's sediment concentration distribution; and $z=$ Rouse number that expresses the ratio of the sediment properties to the hydraulic characteristics of the flow (Julien 1995, p. 185). Eq. (2) comes from the product of the logarithmic velocity profile and Rouse sediment concentration distribution. For the purpose of manipulation, the above two integrals can be rearranged as

$$
J_{1}(z)=\int_{0}^{1}\left(\frac{1-\xi}{\xi}\right)^{z} d \xi-\int_{0}^{E}\left(\frac{1-\xi}{\xi}\right)^{z} d \xi
$$

and

$$
J_{2}(z)=\int_{0}^{1}\left(\frac{1-\xi}{\xi}\right)^{z} \ln \xi d \xi-\int_{0}^{E}\left(\frac{1-\xi}{\xi}\right)^{z} \ln \xi d \xi
$$

\section{Integral $J_{1}$}

After using Beta function, Guo and Hui (1991) and Guo and Wood (1995) found that for $z<1$,

$$
\int_{0}^{1}\left(\frac{1-\xi}{\xi}\right)^{z} d \xi=B(1+z, 1-z)=\frac{\Gamma(1+z) \Gamma(1-z)}{\Gamma(2)}=\frac{z \pi}{\sin z \pi}
$$

On the other hand, the second term on the right-hand side of Eq. (3) is defined as

$$
F_{1}(z)=\int_{0}^{E}\left(\frac{1-\xi}{\xi}\right)^{z} d \xi
$$

It can be solved using integration by parts as

$$
F_{1}(z)=E\left(\frac{1-E}{E}\right)^{z}+z F_{1}(z)+z F_{1}(z-1)
$$




$$
F_{1}(z)=-\frac{1}{z-1} \frac{(1-E)^{z}}{E^{z-1}}-\frac{z}{z-1} F_{1}(z-1)
$$

Multiple applications of the above recurrence formula results in

$$
\begin{aligned}
F_{1}(z) & =-\frac{(1-E)^{z}}{E^{z-1}} \frac{1}{z-1}+\frac{(1-E)^{z-1}}{E^{z-2}} \frac{z}{z-1} \frac{1}{z-2}+\frac{z}{z-2} F_{1}(z-2) \\
& =\frac{(1-E)^{z}}{E^{z-1}}-z \sum_{k=1}^{\infty} \frac{(-1)^{k}}{k-z}\left(\frac{E}{1-E}\right)^{k-z}
\end{aligned}
$$

Thus, from Eqs. (3), (5), (6), and (8), one can get $J_{1}$ for $z<1$

$$
J_{1}(z)=\frac{z \pi}{\sin z \pi}-\underbrace{\left\{\frac{(1-E)^{z}}{E^{z-1}}-z \sum_{k=1}^{\infty} \frac{(-1)^{k}}{k-z}\left(\frac{E}{1-E}\right)^{k-z}\right\}}_{F_{1}(z)}
$$

Similar to Eq. (7b), applying integration by parts to Eq. (1), one gets

$$
J_{1}(z)=\frac{1}{z-1} \frac{(1-E)^{z}}{E^{z-1}}-\frac{z}{z-1} J_{1}(z-1)
$$

Therefore, for $1<z<2$, one obtains

$$
\begin{aligned}
J_{1}(z)= & \frac{1}{z-1} \frac{(1-E)^{z}}{E^{z-1}}-\frac{z}{z-1}\left\{\frac{(z-1) \pi}{\sin (z-1) \pi}-\frac{(1-E)^{z-1}}{E^{z-2}}\right. \\
& \left.+(z-1) \sum_{k=1}^{\infty} \frac{(-1)^{k}}{k-(z-1)}\left(\frac{E}{1-E}\right)^{k-(z-1)}\right\} \\
= & \frac{1}{z-1} \frac{(1-E)^{z}}{E^{z-1}}+\frac{z \pi}{\sin z \pi}+\frac{z}{z-1} \frac{(1-E)^{z-1}}{E^{z-2}} \\
& -z \sum_{k=1}^{\infty} \frac{(-1)^{k}}{k-z+1}\left(\frac{E}{1-E}\right)^{k-z+1} \\
= & \frac{z \pi}{\sin z \pi}-\frac{(1-E)^{z}}{E^{z-1}}+z \sum_{k=1}^{\infty} \frac{(-1)^{k}}{k-z}\left(\frac{E}{1-E}\right)^{k-z}
\end{aligned}
$$

which is identical to Eq. (9). Furthermore, one can recognize the self similarity of Eq. (9) for any noninteger value of $z$.

For any integer $z=n$, a closed solution can be obtained by applying the binomial theorem to the integrand

$$
\begin{aligned}
J_{1}(n)= & \int_{E}^{1}\left(\frac{1-\xi}{\xi}\right)^{n} d \xi=\sum_{k=0}^{n} \frac{(-1)^{k} n !}{(n-k) ! k !} \int_{E}^{1} \xi^{k-n} d \xi \\
= & \sum_{k=0}^{n-2 \geqslant 0} \frac{(-1)^{k} n !}{(n-k) ! k !} \frac{1-E^{k-n+1}}{k-n+1} \\
& +n(-1)^{n-1} \int_{E}^{1} \xi^{-1} d \xi+(-1)^{n} \int_{E}^{1} d \xi \\
& =\sum_{k=0}^{n-2 \geqslant 0} \frac{(-1)^{k} n !}{(n-k) ! k !} \frac{E^{k-n+1}-1}{n-k-1}+(-1)^{n}(n \ln E-E+1)
\end{aligned}
$$

For example, when $n=3$, it gives

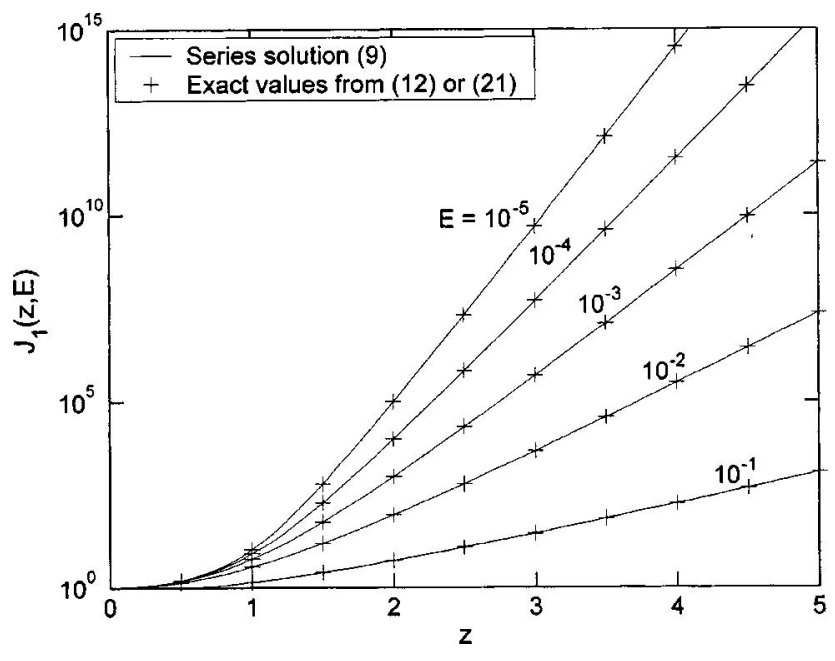

Fig. 1. Plot of integral $J_{1}(z, E)$, Eq. (9)

$$
J_{1}(3)=-3 \ln E+\frac{1}{2 E^{2}}-\frac{3}{E}+\frac{3}{2}+E
$$

To avoid computational overflow, it is suggested to apply Eq. (9) to any noninteger $z$ value, and use Eq. (12) for any integer $z$ value. In practice, an integer $z$ can be considered $z=n \pm 10^{-3}$. For example, if $z=2.998$, Eq. (9) is used; if $z=2.999$, it can be considered $z \approx 3$ and Eq. (12) is then applied. Besides, from Fig. 1, one can see that Eq. (9) converges to Eq. (12) when $z$ tends to an integer $n$. In fact, this convergence can also be analytically demonstrated, the proof being beyond the scope of this note.

\section{Integral $J_{2}$}

Guo and Wood (1995) and Guo (2002) also showed that for $z<1$, one has

$$
\begin{aligned}
\int_{0}^{1}\left(\frac{1-\xi}{\xi}\right) z \ln \xi d \xi & =\frac{z \pi}{\sin z \pi}[\psi(1-z)-(1-\gamma)] \\
& =\frac{z \pi}{\sin z \pi}[\psi(z)+\pi \cot z \pi-(1-\gamma)] \\
& =\frac{z \pi}{\sin z \pi}\left\{\pi \cot z \pi-1-\frac{1}{z}+\sum_{k=1}^{\infty}\left(\frac{1}{k}-\frac{1}{z+k}\right)\right\}
\end{aligned}
$$

where $\gamma=0.577215 \ldots=$ Euler constant; and $\psi(z)=$ psi function, a special function (Andrews 1985). Defining

$$
F_{2}(z)=\int_{0}^{E}\left(\frac{1-\xi}{\xi}\right) \ln \xi d \xi
$$

in Eq. (4) and applying integration by parts gives

$$
F_{2}(z)=E\left(\frac{1-E}{E}\right)^{z} \ln E+z F_{2}(z-1)+z F_{2}(z)-F_{1}(z)
$$




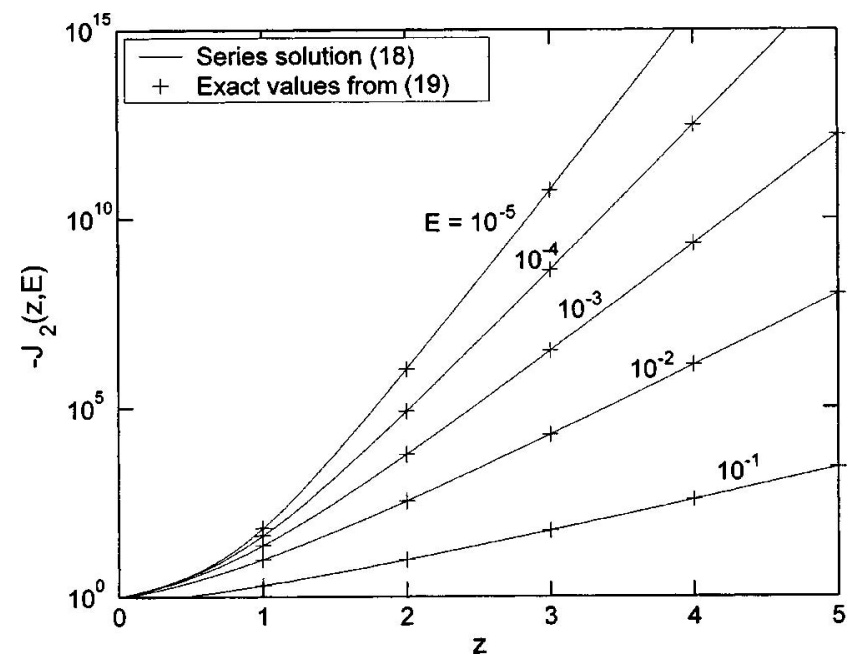

Fig. 2. Plot of integral $J_{2}(z, E)$, Eq. (18)

$$
F_{2}(z)=-\frac{(1-E)^{z}}{E^{z-1}} \frac{\ln E}{z-1}-\frac{z}{(z-1)} F_{2}(z-1)+\frac{F_{1}(z)}{(z-1)}
$$

This result is similar to Eq. (7b). After a complicated derivation, one can show that

$$
F_{2}(z)=F_{1}(z)\left(\ln E+\frac{1}{z-1}\right)+z \sum_{k=1}^{\infty} \frac{(-1)^{k} F_{1}(z-k)}{(z-k)(z-k-1)}
$$

in which $F_{1}(z)$ is estimated by Eq. (8). Finally, Eq. (4) becomes

$$
\begin{aligned}
J_{2}(z)= & \frac{z \pi}{\sin z \pi}\left\{\pi \cot z \pi-1-\frac{1}{z}+\sum_{k=1}^{\infty}\left(\frac{1}{k}-\frac{1}{z+k}\right)\right\} \\
& -\underbrace{\left\{F_{1}(z)\left(\ln E+\frac{1}{z-1}\right)+z \sum_{k=1}^{\infty} \frac{(-1)^{k} F_{1}(z-k)}{(z-k)(z-k-1)}\right\}}_{F_{2}(z)}
\end{aligned}
$$

Like Eq. (9), Eq. (18) is valid for any noninteger $z$ although it is derived for $z<1$. For integer $z=n$, the following closed solution exists

$$
\begin{aligned}
J_{2}(n)= & \int_{E}^{1}\left(\frac{1-\xi}{\xi}\right)^{n} \ln \xi d \xi=\sum_{k=0}^{n} \frac{n !(-1)^{k}}{(n-k) ! k !} \int_{E}^{1} \xi^{k-n} \ln \xi d \xi \\
= & \sum_{k=0}^{n-2 \geqslant 0} \frac{(-1)^{k} n !}{(n-k) ! k !} \int_{E}^{1} \xi^{k-n} \ln \xi d \xi-(-1)^{n} n \int_{E}^{1} \frac{\ln \xi}{\xi} d \xi \\
& +(-1)^{n} \int_{E}^{1} \ln \xi d \xi \\
& =\sum_{k=0}^{n-2 \geqslant 0} \frac{(-1)^{k} n !}{(n-k) ! k !} \cdot\left\{\frac{E^{1+k-n} \ln E}{n-k-1}+\frac{E^{1+k-n}-1}{(n-k-1)^{2}}\right\} \\
& +(-1)^{n}\left\{\frac{n}{2} \ln ^{2} E-E \ln E+E-1\right\}
\end{aligned}
$$

For the interest of application, the convergence of Eq. (18) to Eq. (19) is only shown in Fig. 2.

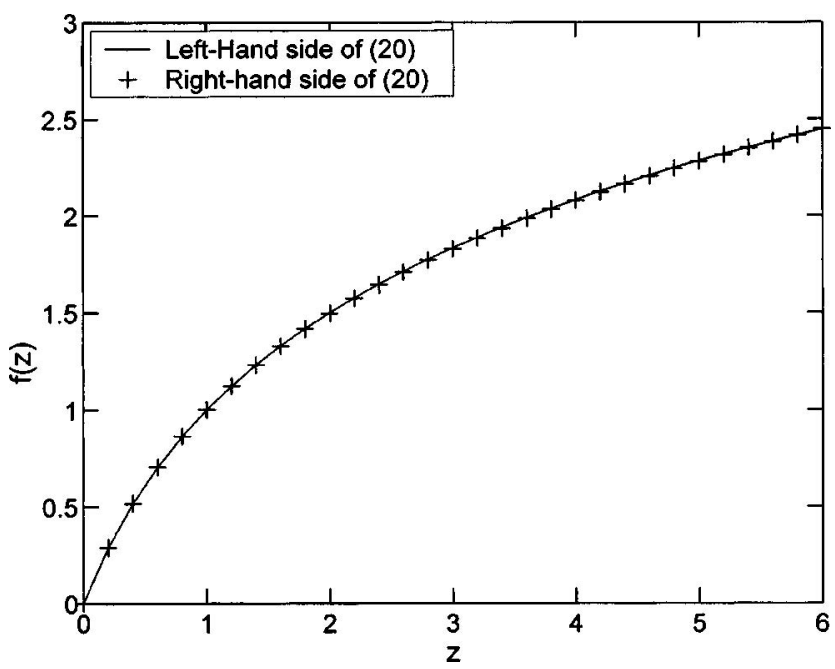

Fig. 3. Approximation of Eq. (20)

\section{Proposed Algorithm and Convergence}

Eqs. (9) and (18) include three infinite series. Series (8) and (17) are rapidly convergent as soon as $k-z>1$, because $E^{k-z}$ quickly tends to zero. In practice, taking the first 10 terms in Eqs. (8) and (17) is accurate enough since there is no sediment transport under $z>10$. The convergence of the first series in Eq. (18) is comparatively slower. For calculation, the following approximation can be used in a program

$$
\sum_{k=1}^{\infty}\left(\frac{1}{k}-\frac{1}{z+k}\right) \equiv f(z) \approx \frac{\pi^{2}}{6} \frac{z}{(1+z)^{0.7162}}
$$

which is shown in Fig. 3 where the maximum relative error is $0.26 \%$ for $0 \leqslant z \leqslant 6$.

The above analysis can be summarized in the form of a computational algorithm. First, for an integer value $z$, i.e., $\mid z$ $-\operatorname{round}(z) \mid<10^{-3}$, Eqs. (12) and (19) are directly applied. Otherwise, the following algorithm is used.

- Step 1: Estimate $F_{1}(z)$ from Eq. (8) using a maximum of 10 terms, $k=10$.

- Step 2: Estimate $J_{1}(z)$ from Eq. (9).

- Step 3: Estimate the first series in Eq. (18) by using the approximation (20).

- Step 4: Estimate $F_{2}(z)$ from Eq. (17) using $k=10$ terms.

- Step 5: Estimate $J_{2}(z)$ from Eq. (18).

A Fortran subroutine or Excel spreadsheet can be downloaded from http://courses.nus.edu.sg/course/cveguoj/ce5309/pierre.html for the above algorithm. The results of applying this algorithm are plotted in Figs. 1 and 2 where the symbol of a cross indicates the exact values from Eqs. (12) and (19). In addition, the exact values of $J_{1}$ for $z=n+1 / 2$ can be found with Maple and are also plotted in Fig. 1. For example,

$$
\begin{gathered}
J_{1}\left(\frac{1}{2}\right)=\frac{\pi}{4}-\frac{1}{2} \sin ^{-1}(2 E-1)-E \sqrt{\frac{1}{E}-1} \\
J_{1}\left(\frac{3}{2}\right)=-\frac{3 \pi}{4}+\frac{3}{2} \sin ^{-1}(2 E-1)+(2+E) \sqrt{\frac{1}{E}-1}
\end{gathered}
$$




$$
\begin{aligned}
J_{1}\left(\frac{5}{2}\right)= & \frac{5 \pi}{4}-\frac{5}{2} \sin ^{-1}(2 E-1)+\left(\frac{2}{3 E}-\frac{14}{3}-E\right) \sqrt{\frac{1}{E}-1} \\
J_{1}\left(\frac{7}{2}\right)= & -\frac{7 \pi}{4}+\frac{7}{2} \sin ^{-1}(2 E-1) \\
& +\left(\frac{2}{5 E^{2}}-\frac{32}{15 E}+\frac{116}{15}+E\right) \sqrt{\frac{1}{E}-1} \\
J_{1}\left(\frac{9}{2}\right)= & \frac{9 \pi}{4}-\frac{9}{2} \sin ^{-1}(2 E-1) \\
& +\left(\frac{2}{7 E^{3}}-\frac{58}{35 E^{2}}+\frac{156}{35 E}-\frac{388}{35}-E\right) \sqrt{\frac{1}{E}-1}
\end{aligned}
$$

One can see that Eqs. (9) and (18), respectively, converge to Eqs. (12) and (19), the results for integer $z$ values from Eq. (21) also coincide with those from Eq. (9). Thus, one can consider that Eqs. (9) and (18) correctly represent the accurate vales of $J_{1}$ and $J_{2}$, respectively. The numerical calculation shows that the presented approximations are computationally efficient and can avoid computational overflow. Therefore, they can be incorporated into professional software like HEC-RAS or HEC-6.

\section{Conclusions}

This note presents an effective approximation to Einstein integrals $J_{1}$ and $J_{2}$ that are valid over the entire range of the Rouse number $z$ and the relative bed-layer thickness $E$. The approximations can be readily implemented using widespread tools such as programmable calculators, spreadsheets, Fortran, or MatLab. In particular, it may provide a simple way to incorporate Einstein bed load function into widely used hydraulic software. The numerical experiment shows that the proposed algorithm rapidly converges to the exact values of $J_{1}$ and $J_{2}$.

\section{References}

Andrews, L. C. (1985). Special functions of mathematics for engineers, McGraw-Hill, New York.

Einstein, H. A. (1950). "The bed load function for sediment transportation in open channel flows." U.S. Department of Agriculture, Soil Conservation Service, Washington, D.C.

Guo, J. (2002). "Approximations of gamma function and psi function and their applications in sediment transport." Advances in hydraulics and water engineering, Proc. 13th IAHR-APD Congress, World Scientific, Singapore, 1, 219-223.

Guo, J., and Hui, Y. J. (1991). "A further study on Einstein's sediment transport theory." Adv. Water Sci., 2(2), 81-91 (in Chinese).

Guo, J., and Wood, W. L. (1995). "Fine suspended sediment transport rates." J. Hydraul. Eng., 121(12), 919-922.

Julien, P. Y. (1995). Erosion and sedimentation, Cambridge University Press, Cambridge, U.K.

Nakato, T. (1984). "Numerical integration of Einstein's integrals, $I_{1}$ and I2." J. Hydraul. Eng., 110(12), 1863-1868.

U.S. Army Corps of Engineers. (1993). HEC-6 user's manual, version 4.1, Hydrologic Engineering Center, Davis, Calif.

U.S. Army Corps of Engineers. (2003). HEC-RAS user's manual, Version 3.1.1, Hydrologic Engineering Center, Davis, Calif. 